Des institutrices pour les garçons. La féminisation de l'enseignement primaire en France, des années 1880 aux années 1920

School Mistresses for Boys. The Feminization of Primary Education in France from the 1880s to the $1920 \mathrm{~s}$

Lehrerinnen in Knabenschulen. Zur Feminisierung des Grundschulwesens in Frankreich zwischen 1880 und 1920

Maestras para los chicos. La feminización de la primera enseñanza en Francia, de los años 1880 a los años 1920

\title{
Jean-François Chanet
}

\section{OpenEdition}

Journals

Édition électronique

URL : https://journals.openedition.org/histoire-education/1425

DOI : 10.4000/histoire-education. 1425

ISSN : 2102-5452

Éditeur

ENS Éditions

Édition imprimée

Date de publication : 1 septembre 2007

Pagination : 171-193

ISBN : 978-2-7342-1101-3

ISSN : 0221-6280

\section{Référence électronique}

Jean-François Chanet, « Des institutrices pour les garçons. La féminisation de l'enseignement

primaire en France, des années 1880 aux années 1920 » Histoire de l'éducation [En ligne], 115-116|

2007, mis en ligne le 01 janvier 2012, consulté le 20 mai 2021. URL : http://journals.openedition.org/ histoire-education/1425; DOI : https://doi.org/10.4000/histoire-education.1425 


\title{
DES INSTITUTRICES POUR LES GARÇONS La féminisation de l'enseignement primaire en France des années 1880 aux années 1920
}

\author{
par Jean-François CHANET
}

L'un des apports les plus importants de Françoise Mayeur à l'histoire de l'éducation en France à l'époque contemporaine est l'analyse qu'elle a donnée de la rencontre, annoncée par Jules Ferry dans son célèbre discours de 1870 à la salle Molière, entre la « sollicitude des républicains pour l'enseignement féminin » et leur « œuvre de laïcité, à la fois plus urgente et plus malaisée » ici qu'ailleurs, en raison de la solidité des positions tenues par l'Église (1). Son analyse a bien fait ressortir la durable contradiction entre une valorisation réelle de la part prise par les femmes à l'effort d'éducation nationale et la persistance d'une conception d'ensemble du système scolaire et de la société où se reflètent largement la position dominante des hommes et l'attachement à son maintien.

Nous ne proposons d'ajouter ici que quelques éléments propres à éclairer cette contradiction. On se rappelle les dispositions générales prévues à l'article 6 de la loi Goblet du 30 octobre 1886: «L'enseignement est donné par des instituteurs dans les écoles de garçons, par des institutrices dans les écoles de filles, dans les écoles maternelles, dans les écoles ou les classes enfantines et dans les écoles mixtes. Dans les écoles de garçons, des femmes peuvent être admises à enseigner à titre d'adjointes, sous la condition d'être épouse, sœur ou parente en ligne directe du directeur de l'école (2). » Dans une récente étude, Pierre Caspard rappelle que cette dernière disposition « énonce, sur le mode prescriptif, ce qu'a été, de facto, la place des

(1) Françoise Mayeur, De la Révolution à l'École républicaine, T. III de LouisHenri Parias (dir.), Histoire générale de l'enseignement et de l'éducation en France, préface de René Rémond, Paris, Nouvelle Librairie de France, 1981, p. 542.

(2) Recueil des lois et actes de l'instruction publique, 1886, n ${ }^{\circ} 43$, pp. 810-811. 
femmes dans l'enseignement assuré par les communes neuchâteloises à la fin de l'Ancien Régime ", et le phénomène n'était pas rare en France à la même époque. Cette constatation - ajoute-t-il - «prête à réfléchir à la pauvreté de la grammaire des rapports de sexes dans l'enseignement, comme à la lenteur de ses transformations, à l'échelle des siècles et des pays (1). » Pour montrer à quel point cela reste vrai dans la France de la III ${ }^{\text {e }}$ République, priorité sera donnée aux sources administratives - et surtout aux rapports annuels des inspecteurs d'académie sur la situation de l'enseignement primaire dans leur département - ainsi qu'aux revues pédagogiques et corporatives, nombreuses, on le sait (2), et, pour autant qu'on puisse en juger, régulièrement lues par le public auquel elles étaient destinées (3).

Nous reviendrons en premier lieu sur la rapidité avec laquelle on est passé, dans les années 1880, du comblement du retard de l'instruction publique laïque pour les filles à l'emploi d'institutrices dans les écoles mixtes et les petites classes des écoles de garçons. Le désordre créé par la guerre n'apparaît dès lors que comme un accélérateur de tendances déjà existantes. L'ampleur et la durée de l'appel exceptionnel fait aux femmes a-t-il du moins permis de donner à leur travail une reconnaissance autre que symbolique ? Oui, à en juger par la loi de 1919 qui établit, pour la première fois en France, l'égalité de traitement entre hommes et femmes dans un service public. Mais ce n'est pas là le seul élément d'appréciation de l'évolution. On continue en effet, longtemps après l'armistice, à voir s'exprimer le souhait d'un « retour à la normale » conçu comme l'improbable rattrapage des positions masculines perdues.

\section{LA RAPIDE ÉVOLUTION DES « BESOINS DU SERVICE »}

À peine installé au ministère de l'Instruction publique, en 1879 , Jules Ferry a donné la priorité au vote de la loi sur les écoles normales de jeunes filles parce qu'il y avait, pour lui comme pour Paul Bert,

(1) Pierre Caspard, «La Maîtresse cachée. Aux origines de l'institutrice publique, 1650-1850 », Annales Pestalozzi: recherches en histoire de l'éducation, $\mathrm{n}^{\circ}$ 3, 20042005, Éducation des filles aux XVII et XIX'e siècles, p. 18.

(2) Voir P. Caspard (dir.), La presse d'éducation et d'enseignement en France, XVIII 'e siècle-1940, répertoire analytique, 4 tomes, Paris, INRP/CNRS, 1981-1991.

(3) Mona Ozouf, Jacques Ozouf, La République des instituteurs, Paris, Gallimard/Le Seuil, 1992, pp. 266-268. 
une particulière urgence à doter tous les départements d'une élite enseignante féminine laïque. Encore celle-ci n'était-elle qu'une avant-garde dont les républicains attendaient qu'elle prît dans la vie scolaire locale un rapide ascendant. Les études récentes sur ces établissements s'accordent sur le caractère rigoureusement normatif et l'austérité du programme imposé aux élèves-maitresses depuis le lever à cinq heures du matin jusqu'au coucher à vingt et une heures trente (1). Toutefois, leurs effectifs ne permettaient pas de remplacer partout le personnel congréganiste, comme la loi Goblet du 30 octobre 1886 en créa l'obligation.

L'article $1^{\text {er }}$ de la loi du 9 août 1879 accordait aux départements un délai de quatre ans pour financer la construction des écoles normales d'institutrices manquantes. Une ligne nord-sud qui sinue entre Rouen, Alençon, Moulins et Perpignan, permet de distinguer, entre les conseils généraux, les plus dociles et les plus réticents (2). À l'échéance prévue, en 1883 , la moitié est du pays est à peu près couverte d'écoles normales de jeunes filles. Il a fallu six ans de plus à la moitié ouest pour rattraper son retard. Dans dix-sept départements, l'ouverture de l'école normale n'a eu lieu qu'en 1884 (Charente, Corrèze, Haute-Garonne, Gironde, Morbihan, Vendée), 1885 (HautesAlpes, Finistère, Meuse) ou 1886 (Basses-Alpes, Calvados, Côtesdu-Nord, Gers, Ille-et-Vilaine, Landes, Maine-et-Loire, Manche); et en 1886, dans huit départements la loi n'avait pas encore reçu d'exécution (Aveyron, Creuse, Eure, Indre, Lot, Mayenne, Tarn, Vienne). La première promotion des élèves-maîtresses de l'Indre ne fut accueillie à l'école de Châteauroux que dix ans après le vote de la loi, à la rentrée de 1889 (3).

(1) Voir notamment Sharif Gemie, Women and Schooling in France, 1815-1914. Gender, Authority and Identity in the Female Schooling Sector, Keele, Keele University Press, 1995, p. 96, et Anne Therese Quartararo, Women Teachers and Popular Education in Nineteenth-Century France: Social Values and Corporate Identity at the Normal School Institution, Cranbury, University of Delaware Press, 1995.

(2) Dans son « Rapport au ministre de l'Intérieur sur les travaux des conseils généraux pendant l'année 1878-1879 », M. de Crisenoy, conseiller d'État, directeur de l'administration départementale et communale, avance les chiffres suivants: sur les 69 conseils généraux auxquels la loi fait obligation de voter la création d'une école normale d'institutrices, 25 « en ont décidé la création immédiate, [...] 33 ont ajourné la question, 9 en ont renvoyé l'examen à la prochaine session. » (Recueil des lois et actes de l'instruction publique, $\left.1879, \mathrm{n}^{\circ} 27, \mathrm{p} .624\right)$.

(3) Bulletin départemental [désormais BD] de l'Indre, août 1889, p. 108. 
Certes, le retard scolaire des départements qui n'ont pas appliqué la loi dans les délais était inégal. Dans ceux, comme la Mayenne, où existait un cours normal, la construction de la nouvelle école pouvait à la rigueur attendre. Mais d'autres ont laissé expirer le délai de quatre ans sans se doter même d'un cours normal: en 1884, le conseil général du Tarn se contentait encore de voter un crédit annuel de trois mille francs pour l'entretien de quelques boursières à l'école congréganiste du Bon-Sauveur à Albi. La construction d'une école normale ne fut décidée qu'en 1888 (1). Compte tenu de cette variété dans les décisions des conseils généraux, on peut comprendre qu'en 1884, la Revue pédagogique ait salué avec des accents de triomphe la construction prochaine de trente écoles normales d'institutrices, " toutes situées aux chefs-lieux des départements, sauf celle de la Manche qui sera à Coutances. [...] Ce résultat s'imposait de par la loi ; il n'en est pas moins à signaler (2). »

L'avance dans l'installation d'écoles normales d'instituteurs n'est au demeurant que l'un des éléments d'appréciation de la primauté masculine dans l'enseignement primaire. Plus anciennes, ces écoles accueillaient aussi des promotions plus nombreuses. En 1884, leur effectif n'était inférieur à cinquante élèves que dans vingt-quatre départements. Ce chiffre peut d'ailleurs être réduit d'une unité, si l'on rapporte les vingt-deux élèves de l'école de Belfort aux dimensions du Territoire. À l'inverse, l'effectif de l'école normale d'institutrices ne dépassait cinquante élèves que dans treize départements - quatorze si l'on y ajoute les Vosges, puisque l'école d'Épinal en comptait tout juste cinquante. On retrouve là l'écart déjà observé entre la France de l'Est et celle de l'Ouest: à l'ouest d'une ligne Rouen-Aix qui passerait par Chartres et Le Puy, aucune école normale de filles n'était fréquentée par plus de cinquante élèves.

(1) Rapport sur la situation de l'enseignement primaire dans le département pendant l'année 1888 [nous indiquerons désormais seulement l'année pour chaque rapport cité], BD Tarn, sept. 1889, pp. 147-148. Voir aussi rapport 1883, sept. 1884, p. 163.

(2) "Les écoles normales primaires en 1884 », Revue pédagogique, 1884, V, p. 145. L'insistance sur la localisation au chef-lieu est conforme au souhait des républicains, en réaction aux quelques créations antérieures à 1879 dans des villes de moindre importance. 
Alors que l'application des textes sur la laïcité, l'obligation et les écoles de hameau (1) entraînait la création d'un grand nombre de postes, il fallait donc faire appel à des brevetées non normaliennes, dans des proportions plus ou moins importantes selon les départements (2). Les administrateurs étaient les premiers conscients que ce n'était pas là le meilleur moyen de garantir la compétence du personnel et son attachement à la laïcité, en un temps où les congrégations assuraient encore une part importante de la scolarisation des filles. Dans le département du Rhône, étudié par Sarah Curtis (3), la moyenne des élèves par institutrice publique était de 30 , ce qui dans l'absolu aurait été satisfaisant si $60 \%$ des filles n'avaient été inscrites dans une école congréganiste. La variété, en ce domaine aussi, doit être soulignée, ne serait-ce que pour démentir l'image d'uniformité trop souvent encore attachée à l'enseignement primaire de la III République. Durant l'année scolaire 1895-1896, l'école normale d'institutrices de Rodez ne recevait que 31 élèves pour un personnel féminin départemental de 826 personnes, soit le même effectif que celle de Châteauroux, alors qu'on ne comptait dans l'Indre que 288 institutrices publiques en exercice. Pour la revue L'Instruction primaire, il était « difficile d'expliquer de semblables différences dans un personnel soumis aux mêmes règles pour le recrutement, les services, l'admission à la retraite et aussi pour la mortalité (4) ».

(1) La loi Ferry du 28 mars 1882 et la loi Goblet du 30 octobre 1886 ne sont que les principaux de ces textes. Par décret du 10 octobre 1881, les écoles primaires qui comptent moins de vingt-cinq élèves seront désormais seules considérées comme écoles de hameau sous la direction d'adjoints ou d'adjointes, ce qui signifie qu'au-dessus de cet effectif, l'instituteur ou l'institutrice seront assimilés à leurs collègues de la ville. La loi du 20 mars 1883, relative à la caisse des écoles, fixe à trois kilomètres la distance maximale aux habitations et à vingt le nombre d'élèves justifiant l'ouverture d'une école.

(2) Rappelons que les femmes se présentent plus nombreuses que les hommes aux deux brevets et qu'à partir de 1873, tous les départements français délivrent plus de brevets à des femmes qu'à des hommes. Raymond Grew, Patrick J. Harrigan, L'école primaire en France au $19^{e}$ siècle: essai d'histoire quantitative, traduit de l'anglais par Marie-Pierre Gaviano, Paris, Éd. de l'EHESS, 2002, p. 191.

(3) Sarah Ann Curtis, L'enseignement au temps des congrégations: le diocèse de Lyon (1801-1905), trad. de Educating the Faithful: Religion, Schooling and Society in Nineteenth-Century France [2000], par Frère Hervé Daniélou, préface de Claude Langlois, Lyon, Presses universitaires de Lyon, 2003.

(4) F. Mutelet, «Les grandes et les petites écoles normales », L'Instruction primaire, 10 sept. 1899, pp. 15-18. 
La Belle Époque, nul ne l'ignore, est celle des écoles de campagne, le moment où la ramification réelle de l'enseignement primaire public s'est étendue dans le pays au plus près du maillage idéal. Mais ce moment est aussi celui de la plus grande amplitude dans l'écart entre le nombre des normaliens et le nombre total des enseignants. La République n'a pas - tant s'en faut - donné pleine réalité au projet de n'installer dans les campagnes que des maîtres initiés à la science pédagogique, recrutés et formés sous le contrôle et en vue du service de l'État. Certains administrateurs n'ont pas manqué de le déplorer: ainsi Émile Prélat, inspecteur d'académie à Bar-le-Duc, estimait-il en 1901 que l'école normale d'institutrices de la Meuse, « par suite de l'insuffisance numérique des treize premières promotions, n' [avait] pu exercer sur l'éducation professionnelle du personnel qu'une action très limitée (1)».

La situation des écoles normales était cependant différente selon qu'il s'agissait des filles ou des garçons, bien que la corrélation entre la mise en application du décret du 18 janvier 1887, qui avait imposé la possession du brevet élémentaire pour pouvoir se présenter au concours d'entrée, et la crise du recrutement dont ces établissements souffraient encore au tournant du siècle fût difficile à contester dans les deux cas. La réforme avait fait baisser de plus de $25 \%$ le nombre des aspirantes, qui était passé, entre 1887 et 1889, de 3722 à 2733, alors que jusque-là la courbe des candidatures avait toujours été ascendante. Mais le plus important n'est pas là. En 1880, on dénombrait dans les écoles normales d'instituteurs 300 élèves-maîtres de plus que de candidates aux concours des écoles normales d'institutrices - respectivement 1578 et 1278 -; neuf ans plus tard, le nombre des candidatures masculines avait chuté et accusait un retard de 318 unités sur celui des candidatures féminines - 2566 au lieu de 2884 -, alors qu'avait été comblé, pour les jeunes filles, le retard dans les créations d'écoles normales. Les observateurs, favorables ou non à l'école publique, n'ont pas manqué de commenter cette crise du recrutement masculin et de proposer des explications mêlant facteurs internes (concours plus difficile pour un salaire faible, surtout en début de carrière, et lenteur de l'avancement) et externes (attractivité plus grande d'autres carrières à niveau de qualification égal, surtout

(1) Émile Prélat, rapport 1899-1900, BD Meuse, juill. 1901, p. 142. 
après la suppression en 1889 de la dispense du service militaire, compensation ancienne à la modicité des traitements) (1).

L'insuffisance du nombre des normaliens a surtout eu pour effet l'accroissement régulier du nombre des brevetées entrées dans la carrière par la «petite porte » et affectées, on le devine, aux postes les moins recherchés. Une étude sur la circonscription de La Mure (Isère), publiée dans la Revue pédagogique en juillet 1913, offre un exemple parmi d'autres de cette tendance. À la question de savoir si l'administration trouve facilement des maitres pour occuper les postes isolés en montagne, l'auteur donne cette réponse significative:

Le recrutement est assuré d'abord par les deux Écoles normales, puis par la nuée de suppléants ou plutôt de suppléantes qui, dans chaque département, cherchent à entrer dans les cadres. [...]

Généralement on reste peu là-haut. Trop peu même, au gré des populations qui protestent contre les déplacements trop fréquents et contre un défilé d'instituteurs, d'institutrices surtout, déterminant des à-coups, des changements de méthodes et d'habitudes qui ne sont pas pour faciliter l'instruction des enfants. Les écoles temporaires voient chaque année de nouvelles maîtresses; nombre d'écoles permanentes également: les maîtresses qui restent deux ou trois ans dans le même village sont l'exception. [...]

Les normaliens pourraient peut-être faire un stage plus long sur les plateaux alpestres, mais ils ont la chance, à peu près complète, de n'y être point envoyés. Ce sont leurs malheureuses collègues qui sont sacrifiées. Cela tient à deux choses: d'abord au grand nombre d'écoles mixtes, ensuite à la pénurie des maîtres (2)...

Cette évolution témoigne donc à la fois de l'insuffisance du nombre des hommes pour répondre aux besoins créés par le maillage scolaire et de l'habitude prise par l'administration d'employer des femmes dans les écoles mixtes, conformément à l'article 6 de la loi Goblet.

L'habitude n'était cependant pas générale. Le conseil départemental de l'enseignement primaire, réorganisé lui aussi par la loi

(1) On nous permettra de renvoyer à notre article: «Vocation et traitement. Réflexions sur la "nature sociale" du métier d'instituteur dans la France de la $\mathrm{III}^{\mathrm{e}}$ République », Revue d'histoire moderne et contemporaine, 47-3, juillet-septembre 2000, pp. 581-603.

(2) A. Coëffé, « L'enseignement primaire en montagne. Étude faite dans la circonscription de La Mure », Revue pédagogique, 15 juill. 1913, pp. 1-19. 
Goblet, pouvait autoriser des instituteurs à exercer dans une classe mixte. Dans la Seine-et-Oise étudiée par René Crozet, le conseil départemental avait, à la fin du siècle, « accordé à la quasi-totalité des communes rurales cette mesure dérogatoire à la loi du 30 octobre 1886 (1) ». Là où le nombre des instituteurs disponibles était suffisant, les conseils municipaux préféraient voir un homme diriger l'école communale à classe unique, parce qu'ils pouvaient lui confier aussi le secrétariat de la mairie, et même s'il leur fallait rémunérer une adjointe pour l'enseignement de la couture aux filles. Aline Fergant, qui vient de prolonger l'étude de René Crozet, le confirme: si, au début du siècle, l'idée progresse dans le corps enseignant que la « coéducation »- l'organisation d'écoles mixtes à deux classes - est préférable à la séparation des sexes, elle est loin de faire l'unanimité dans les conseils municipaux; elle est surtout mise en pratique par ceux qui peuvent disposer d'un couple d'instituteurs pour diriger l'école mixte, la femme restant adjointe quand le mari est titulaire (2).

L'étude de près de six cents dossiers d'instituteurs et institutrices de la Seine-et-Oise entre 1880 et 1914 met en lumière plusieurs traits caractéristiques de l'inégalité des responsabilités entre hommes et femmes. Si un peu moins du tiers des instituteurs qui ont commencé à exercer entre 1882 et 1891 ont terminé leur carrière comme directeurs d'une école à trois classes, cette proportion atteint la moitié chez ceux qui ont commencé entre 1905 et 1914. À l'inverse, si $15 \%$ seulement des institutrices de la première période sont restées adjointes toute leur carrière, c'est le cas pour $50 \%$ de celles dont l'activité a commencé entre 1905 et 1914 - plus nombreuses en chiffres absolus, dans la mesure où l'effectif du personnel féminin a augmenté plus vite que le nombre des écoles de filles. Dans ce département où l'urbanisation progressait rapidement, beaucoup d'entre elles refusaient l'affectation dans une école rurale à une ou deux classes, étape obligée sur la voie de l'avancement, parce qu'elle les aurait éloignées d'un mari fonctionnaire ou employé aux impératifs professionnels duquel elles restaient soumises. C'est ce qui explique aussi que certaines soient restées adjointes pour ne pas s'éloigner d'un mari titulaire (3).

(1) René Crozet, Les instituteurs de Seine-et-Oise vers 1900, préface de Mona Ozouf, postface de Jacques Girault, Saint-Ouen-l'Aumône, Musée départemental de l'éducation, 1991, p. 398.

(2) Aline Fergant, Les instituteurs de Seine-et-Oise (1880-1914), thèse de doctorat d'histoire, sous la direction de J. Girault, université Paris-XIII, 2007, p. 250.

(3) Ibid., pp. 242-251. 
Dans l'Isère comme dans bien d'autres départements ruraux, la situation était sensiblement différente et le souhait des conseils municipaux ne pouvait être aussi facilement satisfait. Dans ceux où les enfants avaient l'habitude d'entendre parler chez eux une langue régionale, le fait d'être soumis, au moins en début de scolarité, à l'autorité d'une institutrice a probablement joué dans le sens de la priorité officiellement donnée à l'apprentissage du français, car, d'une part, au sein des couples, nombre de témoignages attestent que les femmes percevaient avec plus d'acuité que les hommes son utilité sociale, d'autre part, il a été statistiquement démontré que les institutrices connaissaient et pratiquaient les langues régionales dans une proportion inférieure à celle de leurs collègues masculins: ce n'est d'ailleurs pas un hasard si la "méthode directe », théorisée par l'inspecteur Irénée Carré, qui supposait la substitution immédiate et intégrale du français à l'idiome local, a été souvent qualifiée de « méthode maternelle» (1). Mais dans tous les cas, la tendance était à la féminisation du corps enseignant primaire, au moment où l'exécution de la loi du 7 juillet 1904 interdisant l'enseignement aux congrégations augmentait encore les besoins de personnel dans une proportion plus importante pour les femmes que pour les hommes. La Grande Guerre ne devait donc qu'amplifier et accélérer une évolution déjà engagée.

\section{LE TEMPS DES « INTÉRIMAIRES »}

Dès la première année scolaire du temps de guerre, d'anciens détracteurs de l'école laïque, à l'image de Maurice Barrès (2), ont tenu à ajouter à celui des autorités leur hommage à l'esprit de responsabilité des maîtres. Les institutrices y étaient naturellement associées, puisque c'était à elles qu'il revenait d'assurer la relève des combattants. Au moment où Gabriel Hanotaux rédigeait son rapport sur les prix de vertu de l'Académie française, destiné à honorer les « instituteurs de France », trente mille d'entre eux, soit plus de la

(1) On nous permettra de renvoyer à notre thèse, L'école républicaine et les petites patries, préface de Mona Ozouf, Paris, Aubier, 1996, notamment pp. 273-275.

(2) Maurice Barrès, «L'Amitié des Tranchées et l'Amitié des Écoles », Manuel général de l'instruction primaire [désormais MGIP], 29 mai 1915, p. 395. 
moitié de l'effectif total, étaient mobilisés; deux mille déjà étaient morts, huit mille avaient été mis hors de combat (1).

Que désigne le mot d' « intérimaire », apparu avec le régime d'exception du temps de guerre? D'une part, dès août 1914, le ministère a décidé de suspendre les mises à la retraite. Celles-ci ne furent rétablies qu'à partir de janvier 1916, ce qui permit le retour progressif au mouvement régulier du personnel. D'autre part, la circulaire du 18 août 1914 prescrivit de nommer intérimaires dès la rentrée les élèvesmaîtres de troisième voire de deuxième année, «plus aptes à donner l'enseignement aux élèves des écoles primaires que des suppléants pourvus du seul brevet élémentaire (2) ». Cette disposition pouvait d'autant moins suffire que la plupart des normaliens allaient être bientôt mobilisés à leur tour: ainsi dans le Jura, où, sur vingt-trois élèvesmaitres «mobilisés pour le service scolaire (3) » en janvier 1915, neuf seulement restaient disponibles l'été venu. L'administration a fait appel à des enseignants réfugiés, auxquels la circulaire ministérielle du 2 septembre 1914 avait enjoint de se mettre spontanément à sa disposition (4). Il fallut aussi recruter comme intérimaires des jeunes femmes: non pas seulement les normaliennes, qui déjà ne suffisaient pas à renouveler le personnel féminin, mais beaucoup de ces aspirantes brevetées qui se pressaient devant la « petite porte».

(1) « Hommage de l'Académie française aux instituteurs de France. Extrait du rapport de M. Gabriel Hanotaux sur les prix de vertu », BD Deux-Sèvres, 1915, p. 258.

(2) J. Talbert, rapport 1914-1915, BD Gers, 1915, p. 130. Dans le Gers comme dans d'autres départements - mais non dans tous -, ces élèves-maîtres sont restés en fonctions jusqu'aux vacances de Pâques 1915, c'est-à-dire pendant les mois où la fréquentation était la plus régulière, puis ils ont été remplacés par des intérimaires titulaires du brevet élémentaire et sont retournés à l'école normale pour y achever leur formation.

(3) La formule est de l'inspecteur d'académie A. Jeanperrin, dans son rapport 1914-1915, BD Jura, supplément au n 520, déc. 1915, pp. 15-16.

(4) Une note de l'inspecteur d'académie de l'Aube mentionne, en octobre 1914, à côté d'instituteurs belges « qui, exilés de leur patrie, ont accepté [d'enseigner] dans les écoles de la nôtre », « les nombreux instituteurs des départements français envahis qui occupent momentanément la place de ceux des nôtres que la patrie a appelés aux frontières ». Cité par Jean-François Condette, "Les écoles primaires de l'Aube dans la Grande Guerre (1914-1918) », Éducation et engagements. Les acteurs éducatifs entre l'espace local et la nation dans la France des XIXe-XXe siècles, dossier d'HDR, vol. 4, université Paris-IV-Sorbonne, 2007, p. 656. 
Les conditions nouvelles du recrutement et de l'enseignement ont ainsi commencé par accuser des tendances déjà visibles avant 1914. Dans le Jura comme ailleurs, les demandes furent nombreuses :

Toutes les jeunes filles munies du brevet élémentaire et restées sans emploi par suite des événements s'imaginèrent qu'elles seraient immédiatement accueillies dans l'enseignement. Il y eut des désillusions; au moment où la fréquentation régulière donnait à nos écoles son effectif le plus fort 56 intérimaires furent désignées; avec la diminution de la population scolaire, dès le mois d'avril, ce nombre fut ramené à 42 . Le recrutement donna des résultats supérieurs à celui des jeunes gens. [...] Nos intérimaires rendirent d'utiles services; dans quelques communes, pour mieux conserver les traditions et les habitudes, l'intérim fut confié à la femme de l'instituteur mobilisé lorsqu'elle était pourvue des titres réglementaires (1).

On ne manquera pas de relever que l' « intérim », conforme à une pratique dont on connaît l'ancienneté, est présenté ici comme une utile concession aux «traditions » et aux « habitudes ». Pour cet administrateur du temps de guerre, la mesure est dictée à la fois par une situation d'urgence et par le souci de l'adaptation aux convenances locales. Le pragmatisme affiché reste déterminé et limité par l'attachement à l'étroite « grammaire des rapports de sexes » analysée par Pierre Caspard.

Très vite, l'importance des besoins et l'abondance des candidatures ont incité les inspecteurs d'académie à mettre en garde les prétendantes contre un double risque: celui d'une situation précaire et celui de la facilité qui les conduisait à s'en contenter plutôt que d'assurer leur avenir en essayant d'entrer à l'école normale. Le cas de la Manche est assez caractéristique. Le nombre des petites communes, donc des écoles rurales mixtes, ajouté à l'habitude prise de nommer des adjointes dans les petites classes des écoles de garçons, expliquait que fussent maintenus à l'école normale de Coutances des effectifs plus élevés que dans celles de départements plus peuplés. Or, après l'entrée en guerre, le nombre des aspirantes au concours diminua sensiblement. Pour l'inspecteur d'académie Léon Dériès - qui a dirigé le corps enseignant du département de 1892 à 1923 -, l'explication était toute simple: "C'est seulement que beaucoup de jeunes filles ont demandé et obtenu des emplois d'intérimaires. L'octroi aisé de ces fonctions temporaires a entretenu chez elles des

(1) A. Jeanperrin, rapport 1914-1915, loc. cit. 
illusions dangereuses. Le congédiement nécessaire d'une cinquantaine d'entre elles en octobre dernier [1917], lors de la rentrée des sursitaires, les fera réfléchir et ramènera à l'École Normale des jeunes filles qui s'en détournaient imprudemment (1). »

Qui pourrait prétendre que pareille condition fût un progrès par rapport à celle de l'institutrice d'avant-guerre? «L'institutrice sent que son rôle public la désigne particulièrement à l'attention et que, dans son deuil même, elle doit mettre de la dignité (2)»: tel était, selon Paul Lapie, directeur de l'enseignement primaire au ministère, la clef de la considération nouvelle dont elle devait bénéficier. Revalorisation toute morale, et assortie d'une exigence accrue, en continuité bien plus qu'en rupture avec les « traditions » et les « habitudes » d'avant-guerre: combien d'institutrices ont dû, en plus de leurs tâches ordinaires, ouvrir des garderies pendant les vacances, pratiquer assidûment la couture et le tricot, travailler le jardin, élever des poules et des lapins, quand il ne leur fallait pas remplacer mari ou collègue au secrétariat de la mairie? Pour des maîtresses prévenues qu'elles seraient placées sous le regard pas toujours bienveillant des élus locaux et des parents d'élèves, l'austérité de mœurs, l'exemplarité de conduite n'avaient rien de nouveau, si ce n'est le surcroît d'esprit de sacrifice demandé.

Certains responsables n'ont pas manqué de regretter l'emploi de ces jeunes femmes sans formation et parfois sans autorité sur les garçons qui leur étaient confiés. "Combien d'institutrices éloignées de leur milieu scolaire habituel se sont épuisées en vains efforts! », se

(1) Léon Dériès, rapport 1917, BD Manche, juillet-août 1918, p. 154. Outre la longévité à son poste, qui a justifié l'attribution de son nom à une rue de Saint-Lô, Léon Dériès se distingue par l'abondance de ses publications dans des domaines très variés, allant de l'érudition locale à la fiction édifiante ou à l'essai. Son collègue de la Saône-et-Loire pose au même moment un diagnostic analogue: A. Audran, rapport 1916-1917, Mâcon, 6 juillet 1917, p. 305. Toutefois, précise l'inspecteur d'académie des Vosges à la lumière des chiffres de son département, " le fléchissement a commencé en 1914. Il vient surtout de la facilité avec laquelle beaucoup de jeunes filles, munies du brevet supérieur, obtiennent d'être placées avantageusement dans un département où le nombre des normaliennes ne représente pas tout à fait la moitié du nombre d'institutrices nécessaires. » (E. Meyer, rapport 1917, BD Vosges, 1918, n 618, p. 603).

(2) Paul Lapie reprenait ici à son compte l'affirmation du directeur de l'école normale de Vannes, chargé d'assurer l'intérim de l'inspecteur d'académie du Morbihan, mobilisé (L'Instituteur et la Guerre, articles et documents, Revue pédagogique, janv.fév. 1915, rééd. Paris, Didier, coll. « La Guerre et l'École », 1915, p. 74). 
lamente en 1917 Denis Ginoux, inspecteur d'académie à SaintÉtienne. «À plus forte raison en a-t-il été ainsi pour les intérimaires, dont quelques-unes sont des débutantes (1). » Ce type de reproche a fait dire à Populo - nom de plume de l'inspecteur primaire Émile Salé (2) -, dans la Revue de l'enseignement primaire, qu' " au lieu de casser du sucre sur la tête des intérimaires, on ferait mieux de leur en mettre un petit morceau dans leur café; qu'on ferait mieux aussi, dans l'intérêt de l'enseignement, de les faire circuler un peu moins sur les grandes routes. » Populo a évoqué en termes émouvants, mais avec la conviction que le « retour à la normale » supposait le rétablissement de l'autorité masculine, les drames obscurs du provisoire qui a trop duré:

Certes, quand elles ont pris du service en 1914, elles pensaient - que ceux qui ont vu plus clair leur jettent la première pierre - offrir un dévouement provisoire. Mais il y a de cela trois ans. Tout doucement, elles ont pris goût au métier. Quelques-unes [...] ont préparé l'examen du CAP, l'ont subi avec succès ou sont à la veille de le subir. Nombre d'apprenties sont devenues des maîtresses de valeur. Je vois d'ici une jeune femme qui, ayant pris la direction d'une école mixte abandonnée depuis dix-huit mois, apprit à lire en moins de six mois à vingt-deux enfants de six à huit ans, tout en préparant quelques autres à l'examen du certificat d'études. [...] Elle arrivait à sa classe le matin à bicyclette, mangeait à midi, sur un bout de table dans la classe, et repartait le soir pour le village voisin. Elle arrivait souvent de nuit. Le soleil d'hiver se couche tôt... Si le maître mobilisé revient à son école, la bonne et brave institutrice que je viens de vous montrer n'a plus qu'à rentrer chez elle. On ne lui doit rien. Elle a travaillé. Elle a été payée à raison de 100 francs par mois. Que peut-elle réclamer?

[On] ne peut pourtant pas dire aux maitres [...] de retourner à leurs occupations militaires. Ils ont fait leur devoir de soldats; ils vont faire leur devoir d'instituteurs. C'est l'ordre qui revient à l'école et aussi le savoir avec l'autorité de l'expérience. [...] Et nul ne doit s'en plaindre, pas même les intérimaires.

Pourtant on ne peut pas demander à ces institutrices dépossédées de pousser des cris de joie. Elles avaient l'orgueil de gagner leur pain. Il va

(1) Denis Ginoux, rapport 1916-1917, BD Loire, octobre 1917, p. 186.

(2) Pour les lecteurs de la Revue de l'enseignement primaire, Émile Salé (18471928) a été de 1902 à sa mort l'oncle Populo, qui signait chaque semaine la « Causerie pédagogique ». D'abord instituteur dans l'Yonne, puis inspecteur primaire, à Die puis à Montélimar, il avait fini sa carrière à Château-Thierry et, après avoir repris du service à ce poste pendant la guerre, il s'est retiré au Châtel-Censoir. À ses obsèques, M. Hénon, inspecteur primaire à Avallon, rendit hommage à ce "Claude Tillier de la pédagogie », au « tempérament bourguignon et voltairien, malicieux et caustique, généreux et vaillant » (Revue de l'enseignement primaire, 22 juillet 1928, pp. 499-500). 
falloir en rabattre: elles vont retomber à la charge de leurs familles en attendant qu'elles aient trouvé un autre débouché... à moins qu'elles ne tombent dans la misère...

Peut-on arracher de sa conscience et de son cœur le souvenir des services qu'elles ont rendus? [...] Personnellement, j'en ai vu quelques-unes à l'œuvre, et je n'oublierai de ma vie leur dévouement et leur vaillance. Voulez-vous que je vous dise à l'oreille que plusieurs ont fait le secrétariat de la mairie pour en laisser le bénéfice au titulaire? que quelquesunes ont refusé le supplément communal que leur offrait le conseil municipal au détriment du mobilisé ? Dame ! cela ne s'est pas passé aussi gentiment dans toutes les communes de France. Il y a eu des conflits. Mais, en général, nos intérimaires ont fait largement leur devoir et montré un véritable esprit de solidarité.

Je cherche comment on pourrait le reconnaître autrement que par des fleurs de rhétorique. Je cherche et je ne trouve pas (1).

Comme celle du rapport de Léon Dériès, la date de publication de cet article, peu après la rentrée de 1917, suggère que c'était alors une question d'actualité. En octobre 1916 puis en avril 1917, le gouvernement avait libéré de leurs obligations militaires un certain nombre d'instituteurs appartenant à la réserve de l'armée territoriale et classés dans le service auxiliaire. À la rentrée d'octobre, tous ceux des classes 1888 à 1892, et ceux des classes 1893 à 1903 attachés au service auxiliaire, devaient être rendus à l'administration de l'instruction publique (2). Mais s'il s'est efforcé de concilier les « besoins du service » avec les intérêts de la défense nationale, le ministère s'est dispensé de fixer, pour l'avenir des «intérimaires de guerre », des règles applicables dans l'ensemble du pays. Il a préféré s'en remettre à ses représentants dans les départements, c'est-à-dire aux inspecteurs d'académie. De là une variété certaine dans les mesures adoptées.

\section{L'IMPOSSIBLE « RATTRAPAGE » D'APRÈS-GUERRE}

Ce qui était considéré avant 1914 comme un expédient face à la crise du recrutement masculin, puis, à partir de la mobilisation, comme un moyen de pallier l'absence temporaire des maîtres, apparaît peu à peu, après 1920, comme un remède à leur décimation et une solution durable à la surabondance des candidatures féminines. Le

(1) Populo, « Nos petites intérimaires », Revue de l'enseignement primaire, 25 nov. 1917 , pp. $42-43$.

(2) Voir, parmi d'autres, BD Hérault, août 1917, pp. 136-137. 
ministre a fourni une statistique des pertes publiée au Journal officiel du 19 janvier 1921 : sur 34480 instituteurs mobilisés, 28309 (82,1\%) l'ont été dans les formations combattantes et 6171 (17,9\%) dans les formations de l'arrière; le nombre de ceux qui ont été tués au combat ou qui sont morts des suites de la guerre s'élève à 7407 , soit $21,5 \%$ du total des mobilisés et $26,2 \%$ du total de ceux qui sont allés au front; le nombre des blessés est de 9624 , soit respectivement $27,9 \%$ et $34 \%$ de ces deux totaux (1). Encore faudrait-il ajouter à cette statistique les normaliens en cours de scolarité. Selon un article paru en 1921 dans la revue L'École et la Vie, on doit compter « une moyenne par école de 25 élèves-maitres tombés au champ d'honneur », ce qui obligerait - mais sans doute est-ce là un maximum - à ajouter 2200 morts aux 7400 recensés par le ministre (2).

La gravité même de ce bilan risquait de faire oublier aux autorités que la tendance à l'augmentation de la part des femmes dans le corps enseignant primaire s'inscrivait dans une durée plus longue, et que des mesures administratives ou financières ne pourraient suffire à assurer le rééquilibrage espéré. Dans le Nord, en 1920-1921, l'effectif du personnel en exercice s'élève à 5008 , soit 1865 maîtres et 3143 maîtresses (y compris celles des écoles maternelles). On compte parmi les maîtres 1751 titulaires, 109 stagiaires et 5 intérimaires, parmi les maitresses 2998 titulaires, 95 stagiaires et 50 intérimaires. En 1909, le nombre des instituteurs publics était certes de 2159 (790 titulaires et 1369 adjoints), mais celui des institutrices était déjà de 2200 (758 titulaires et 1442 adjointes) (3). De même, au premier jour scolaire de décembre 1912, le département de la Meurthe-et-Moselle comptait 704 instituteurs et 938 institutrices; en décembre 1919, ces chiffres étaient respectivement de 577 et 954 . « On sait trop pourquoi le nombre des instituteurs a fléchi [...], et il faudra du temps avant qu'ils aient pu être remplacés », commentait l'inspecteur d'académie, sans penser que ce nombre, avant la guerre, était déjà inférieur à celui des institutrices (4).

(1) « Réponses du ministre aux questions écrites », Bulletin administratif, 1921, $\mathrm{n}^{\circ} 2459$, p. 109.

(2) A. Chambord, «Une statistique éloquente », L'École et la Vie, 14 mai 1921, p. 531. Voir aussi le bilan dressé par J.-F. Condette dans « Les écoles primaires de l'Aube dans la Grande Guerre », op. cit., pp. 652-654.

(3) É. Prélat, rapport 1909, BD Nord, sept.-oct. 1910, p. 272, et P. Capra, rapport 1920-1921, p. 360.

(4) Charles Dessez, rapport 1919-1920, BD Meurthe-et-Moselle, 1920, n 7, p. 255. 
Rares étaient ceux qui avaient prévu cette situation, plus rares encore, à cette date, ceux qu'elle n'inquiétait pas. L'inspecteur primaire de Pontivy était de ceux-là. En 1909-1910 étaient en poste dans cette circonscription 111 institutrices et 106 instituteurs publics (1). En 1916-1917, on ne dénombrait plus que 50 instituteurs pour 168 institutrices, dont 59 exerçaient dans des écoles mixtes et 36 dans des écoles de garçons. Voici en quels termes il commentait la situation:

Il est à noter: $1^{\circ}$ Que les écoles mixtes, quand elles fonctionnent dans des hameaux, sont toujours très peuplées; $2^{\circ}$ que dans les écoles devenues mixtes, l'élément masculin est dominant (Saint-Gonnery: 69 garçons et 16 filles; Porcaro : 60 garçons et 6 filles; Saint-Malo-des-Trois-Fontaines : 77 garçons et 3 filles), ce qui rend ces écoles aussi difficiles à diriger que des écoles de garçons.

Or, la plupart des institutrices qui m'ont paru le mieux réussir sont celles qui ont senti leur responsabilité engagée lorsqu'on leur a confié la direction d'une classe; en général, leur fermeté m'a donné toute satisfaction.

Si j'insiste sur cette observation, ce n'est certes pas pour amoindrir l'œuvre accomplie par les instituteurs restés à leurs postes [...], c'est parce qu'il y a à prévoir les lendemains de la guerre. Il m'a paru que cette troisième année de guerre dissipe les angoisses qu'avait pu faire naître d'abord une organisation de fortune et un appel imprévu aux forces féminines. [...] Il est tout à fait permis d'espérer que la progression ira en s'accentuant, et que l'on pourra compter à l'avenir sur cet apport nouveau d'activités féminines (2).

Mais cette position ressemble assez à l'exception qui confirme la règle. $\mathrm{La}$ «progression » rendue nécessaire par le déficit persistant du recrutement masculin inspirait à une majorité d'inspecteurs plus de regret que d'espoir. Quant à la continuité du discours de la presse pédagogique à ce sujet entre l'avant et l'après-guerre, elle n'est guère surprenante, puisque, pour l'essentiel, ce sont les mêmes auteurs qui continuent de s'y exprimer (3). Pour Ferdinand Buisson comme pour beaucoup d'autres, la première cause du mal demeurait l'insuffisance des traitements. L'ancien directeur de l'enseignement primaire n'avait

(1) Cette circonscription était d'ailleurs la seule du département où il ne subsistât aucune congréganiste dans le corps enseignant public. E. Malard, rapport 1909-1910, BD Morbihan, 1910, $\mathrm{n}^{\circ}$ 6, p. 242.

(2) Cité par F. Henry, rapport 1916-1917, BD Morbihan, juill.-août 1917, pp. 172-173.

(3) Voir Michèle Zancarini-Fournel, "Coéducation, gémination, co-instruction, mixité : débats dans l'Éducation nationale (1882-1976) », in Rebecca Rogers (dir.), La mixité dans l'éducation. Enjeux passés et présents, préface de Geneviève Fraisse, Lyon, ENS Éditions, 2004. 
pas hésité à titrer « Traitements d'instituteurs, traitements de famine » son article de rentrée du Manuel général en septembre 1918 (1). La doléance s'appliquait aussi bien aux institutrices qu'aux instituteurs. Dès 1916, son collaborateur André Balz avait attiré l'attention sur le sort de celles qui, sorties de l'école normale au moment de la déclaration de guerre, avaient été « envoyées, comme intérimaires, à la place des instituteurs mobilisés »: « À part quelques exceptions, ces institutrices qui ont le brevet supérieur et le CAP ne touchent que 100 francs par mois, alors que le traitement de leur classe serait de 1600 francs si, au lieu d'être remplaçantes, elles occupaient un poste vacant. [...] Il y a dans les usines des femmes qui gagnent en quelques jours, à tourner des obus, ce que les intérimaires ne gagnent pas en un mois (2). » L'École et la Vie reprenait la même doléance à l'été 1918: "Telle jeune fille qui, à sa sortie de l'école normale, ne reçoit que 1300 francs par an, trouverait très facilement, comme dactylo-sténographe, une place de 150, 180 ou 200 francs par mois, sans avoir eu à préparer des examens longs et difficiles. » L'heure était au pessimisme: « Dès que nos jeunes filles ne trouveront plus dans l'enseignement une carrière suffisamment rémunératrice, elles déserteront, comme le font depuis longtemps les jeunes garçons, nos écoles normales primaires (3). »

Certes, la cherté de la vie à la fin de la guerre donnait quelque légitimité, sinon à ces prévisions alarmistes, du moins aux revendications du personnel enseignant. Mais si les jeunes filles se détournaient des écoles normales en raison de l'insuffisance des traitements, comment expliquer l'encombrement général de l'accès à la carrière? On ne semble pas relever cette contradiction dans la presse de l'époque,

(1) Ferdinand Buisson, « Traitements d'instituteurs, traitements de famine », MGIP, 28 sept. 1918, pp. 3-4.

(2) André Balz, «La Province réclame », MGIP, 18 nov. 1916, p. 116. André Balz était le pseudonyme de Léon Mention (1845-1931). Ce dernier n'était pas un primaire, mais un agrégé d'histoire et un docteur ès lettres, professeur au lycée Charlemagne puis au lycée Henri IV. Il était l'auteur d'un important ouvrage sur L'armée de l'ancien régime, de Louis XIV à la Révolution, qui lui avait valu d'occuper, de 1890 à 1905, la présidence de la commission d'entrée à l'école de Saint-Cyr. Entré au Manuel général en 1899 et bientôt titulaire attitré d'une chronique intitulée « Mon franc-parler », il y fut pendant trente-deux ans un défenseur exigeant des intérêts du personnel primaire et un sévère censeur de l'administration. Son influence rayonna aussi pendant le premier quart du siècle sur l'Association de la Presse d'Enseignement. Voir sa nécrologie dans le MGIP, 16 janv. 1932, pp. 345-346.

(3) M. Jeannard: « Aurons-nous des instituteurs demain? ", L'École et la Vie, 3 août 1918, p. 747. Le même auteur reprend les mêmes arguments dans un article intitulé « Le recrutement des écoles normales », MGIP, 22 oct. 1921, p. 65. 
ce qui étonne d'autant plus que, pour l'ensemble de la France, le nombre des candidates aux concours d'entrée des écoles normales est passé de 6252 en 1913 à 6788 en 1916. Dans le même laps de temps, le nombre des candidats est passé, lui, de 3486 à 3246, petite baisse compensée par la hausse du nombre des admis : 1475 en 1913, 1886 en 1915, 1586 en 1916. André Balz est l'un des rares à reconnaître qu'en dépit des années de guerre, les écoles normales n'ont pas « souffert dans leur recrutement autant qu' on pouvait le craindre et qu'on l'a quelquefois affirmé sans preuves (1) ». Enfin et surtout, les traitements ont été relevés et uniformisés pour les instituteurs et les institutrices en 1919: les stagiaires devaient gagner désormais 3600 francs, la sixième classe 4000 francs, la cinquième 4500, la quatrième 5000 , la troisième 5500 , la deuxième 6000 , la première 6500 et la classe exceptionnelle 7000 francs par an (2).

L'usage qui a été fait par les couples d'instituteurs d'un autre texte important de l'immédiat après-guerre, la loi Roustan du 30 décembre 1921 relative au rapprochement des conjoints dans la fonction publique (3), fournit des indications convergentes. Cette loi a eu pour effet de renforcer la tendance aux mariages entre instituteurs et institutrices et d'augmenter la proportion des postes d'adjoints occupés par des femmes dans les écoles de garçons, selon un processus déjà observé dans la Seine-et-Oise avant 1914. Pour accroître la chance d'obtenir le « poste double » dans la même commune, beaucoup ont dû accepter une séparation, plus moins longue suivant l'encombrement et les besoins dans le département (4). L'acceptation d'un premier poste éloigné de celui du conjoint assurait à l'expatrié(e) en même temps l'accès à la carrière et la promesse du retour. La distance pouvait n'être pas considérable: c'est au titre de l'article 5, relatif au rapprochement à l'intérieur d'un même département, qu'un nombre croissant de couples demandent à bénéficier de

(1) A. Balz, « Le recrutement par l'école normale », MGIP, 14 juill. 1917, p. 656.

(2) « Tableau des nouveaux traitements », L'École et la Vie, 18 oct. 1919, p. 64.

(3) «Article premier. - Dans toute administration, lorsqu'il a été satisfait aux lois sur les emplois réservés, $25 \%$ des postes vacants, au cours de l'année, dans chaque département, sont réservés aux fonctionnaires qui, étrangers au département, sont unis par le mariage, soit à des fonctionnaires du département, soit à des personnes qui y ont fixé depuis plus d'un an leur résidence. » (BD Maine-et-Loire, 1922, pp. 617-618).

(4) Ainsi dans les Bouches-du-Rhône, où il restait beaucoup d'intérimaires à placer: J. Boucher, rapport 1924, p. 193. 
la loi (1). Mais on sait ce qu'étaient encore, dans la France de ce temps-là, les conditions de circulation d'un arrondissement à un autre, voire d'un canton à un autre.

Dans le Manuel général du 30 mars 1929, Louis Schwartz, chef de bureau au ministère, confirme que, « dans ces dernières années, le nombre des ménages d'instituteurs et d'institutrices a notablement augmenté et, en tout cas, dans une proportion supérieure à celle des postes doubles. Pour rapprocher les ménages, il ne s'offrait bien souvent pas d'autre solution que de nommer la femme comme adjointe à l'école où le mari exerce en qualité de directeur ou d'adjoint (2). » Effet plutôt que cause de la diminution des effectifs masculins, la fréquence avec laquelle la « solution » était utilisée permettait toutefois à l'auteur de mettre l'accent sur la « régularité inquiétante » de cette diminution. De 45641 en 1923, le nombre des instituteurs était descendu en 1927 à 42641, puis en 1929 à 41661 , « chiffre le plus bas atteint jusqu'ici (3) ». On devine que ce n'est pas l'augmentation du nombre des classes de filles qui explique l'évolution, mais bien celle du nombre des postes d'adjoints occupés par des institutrices dans les écoles de garçons. $28 \%$ de ces postes étaient dans ce cas en 1925, $29 \%$ en 1928, $30 \%$ en 1929. Ce que Louis Schwartz considérait comme une « crise » présentait cependant des variations sensibles selon les départements, comme le montre la carte établie à partir de ses statistiques.

La différence entre départements alpins et pyrénéens se remarque aussitôt, sans qu' on sache ce qui, des « traditions », des « habitudes », des conséquences démographiques de la guerre ou de l'évolution économique dans les deux régions, contribue le plus à l'explication, ici de l'abondance, là de la rareté des institutrices dans les écoles de garçons. Mais cette incertitude est la même en toutes régions, et il y a là un objet d'étude qui mériterait l'attention des historiens. La situation

(1) « Art. 5. - Dans l'intérieur de chaque département, les autorités administratives se concerteront pour offrir aux ménages de fonctionnaires, aussitôt que l'occasion s'en présentera, sans léser les droits des tiers, soit un poste double, soit deux postes situés dans un même canton. »

(2) Louis Schwartz, « La crise du recrutement masculin », MGIP, 30 mars 1929, pp. 549-550.

(3) Ibid. On notera que les départements d'Alsace et de Lorraine réintégrés après 1918 ne sont pas pris en compte dans ces chiffres. C'est pourquoi ils ne figurent pas sur la carte. Ils se distinguaient par la dualité de provenance du personnel et donc par les distinctions que celle-ci imposait entre le «cadre local » et le «cadre de l'intérieur ». 
Taux d'occupation des postes d'adjoints dans les écoles de garçons par des institutrices en 1929

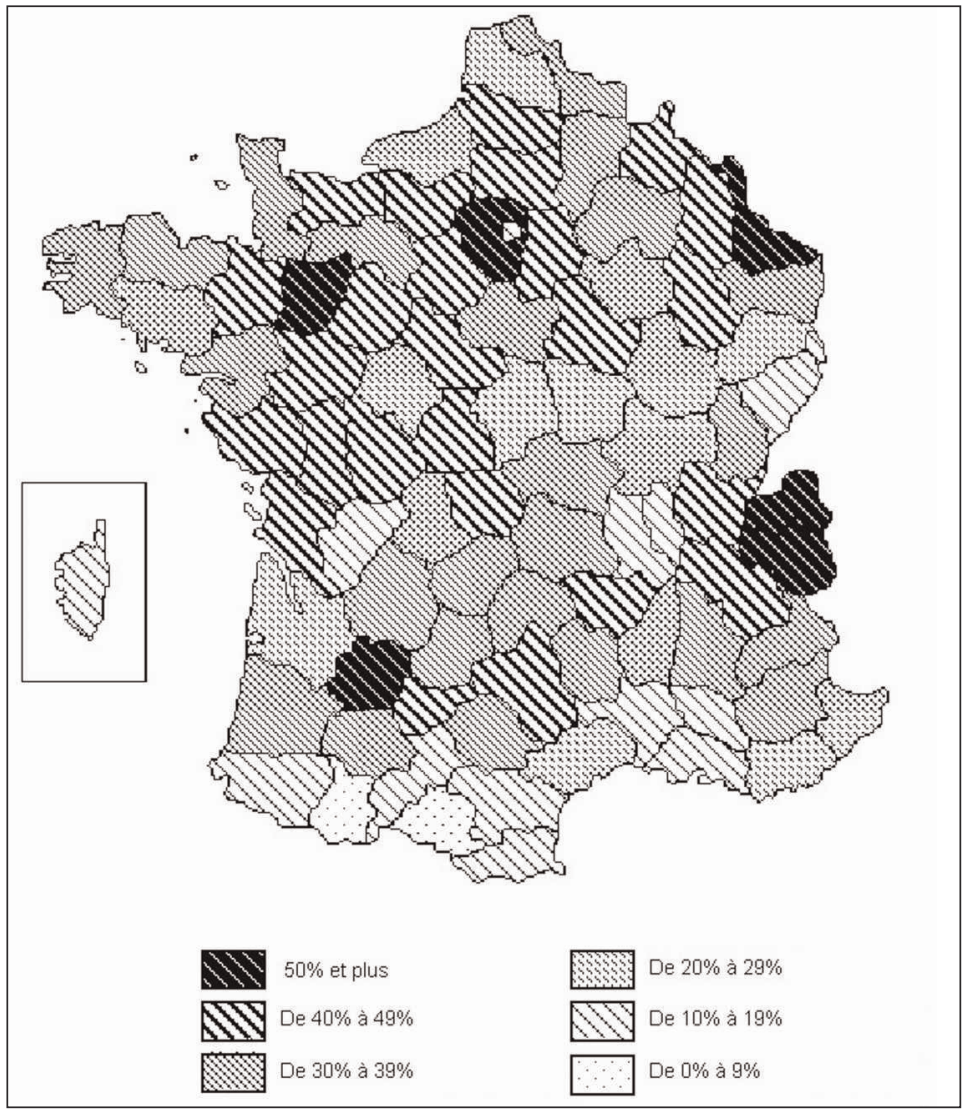

dans la Seine, le Rhône, les Bouches-du-Rhône ou la Haute-Garonne confirme l'attraction des grandes villes. Le personnel masculin y demeure en proportion plus nombreux que dans tous les départements situés au nord d'une ligne qui relierait La Rochelle à Belfort, quels qu'y soient les traits dominants de l'activité économique. Le contraste entre la Seine et les départements limitrophes peut s'expliquer par le caractère régional du recrutement du personnel masculin de la capitale. Mais à partir de quels critères comparer la Mayenne et la Meurthe-et-Moselle? Du moins leur présence dans la même catégorie laisse-t-elle à penser que l'industrialisation et l'urbanisation 
n'ont pas eu partout la même influence sur les conditions de recrutement du personnel masculin.

Ce qu'il nous importait de souligner ici, c'est avant tout le refus des autorités de considérer le processus de féminisation du corps enseignant primaire comme un phénomène durable, sinon irréversible. Édouard Herriot, ministre de l'Instruction publique dans le cabinet Poincaré, a jugé bon d'adresser aux inspecteurs d'académie, le 8 mars 1928, une circulaire où il les appelle à une application plus stricte de la loi: «Les femmes ne doivent enseigner qu'à titre tout à fait exceptionnel dans les écoles de garçons (1) ». Un an après, Louis Schwartz s'inquiète de ce que, la Charente, la Loire, le Rhône et le Doubs mis à part, «tous les départements restés à l'abri de la vague féminine se groupent dans l'extrême midi ». On se demande comment les lectrices du Manuel général ont apprécié ce commentaire.

La réticence de principe à faire grossir cette «vague » est l'une des raisons qui expliquent que la reconnaissance légale de la gémination ait demandé encore un peu de temps. Le mot "gémination », emprunté au vocabulaire des sciences naturelles - Littré le définit comme l' « état de ce qui est disposé par paires »- a été préféré au néologisme de «mixité », absent des dictionnaires du XIX ${ }^{\mathrm{e}}$ siècle, en raison de l'équivoque possible avec la prise en compte de l'appartenance religieuse. Après plus de sept ans de débat, le vote décisif a eu lieu le 9 février 1933 à la Chambre, où il s'est trouvé une majorité pour passer outre à la recommandation négative du Conseil d'État et élargir la catégorie des postes accessibles aux femmes (2). Le rapporteur du projet, Robert, député radical-socialiste de Bar-sur-Aube, en résumait ainsi l'exposé des motifs: « 32000 écoles publiques sont actuellement mixtes; grouper les enfants non d'après le sexe, mais d'après l'âge, voilà le principe; les petits à l'institutrice, les grands à l'instituteur, tel est le système des classes géminées (3)». Nécessité fait loi, mais la hiérarchie des emplois persiste, conforme à l'idée

(1) Ibid., de même que la citation suivante.

(2) Outre son article déjà cité, voir, de Michèle Zancarini-Fournel, « La mixité scolaire: une histoire à faire? », Administration et éducation, 2003, n 1 , pp. 125-132, puis, sous sa direction et celle de Françoise Thébaud, "Coéducation et mixité », Clio. Histoire, femmes et société, $\mathrm{n}^{\circ} 18,2003$.

(3) «Le vote de la gémination », Journal des Instituteurs, 4 mars 1933, p. 350 (de même pour la citation suivante). Louis Marin, député de Nancy - donc représentant d'un département où le déséquilibre entre la proportion des maîtres et celle des 
tenace selon laquelle, au cours moyen, les vertus viriles ne se peuvent bien acquérir que sous l'autorité d'un maître. Peu après, du reste, les suppressions de postes imposées par les décrets-lois de 1934 et 1935 viendront démentir l'espoir de voir augmenter les effectifs des écoles normales d'instituteurs en proportion des « besoins » supposés dans les écoles de garçons.

«Les fonctionnaires de la première génération ont pu contempler la Terre promise de l'égalité avec les hommes; elles n'ont pas toujours eu l'amertume d'en voir les pièges et les ambiguïtés (1). » Cette observation de Françoise Mayeur dans la conclusion de sa thèse vaut aussi bien pour l'enseignement primaire que pour l'enseignement secondaire. D'autres historiennes, Anne-Marie Sohn en premier lieu (2), puis Mona Ozouf (3), Christine Bard (4) ou Siân Reynolds (5), ont à juste titre insisté sur l'influence qu'ont eue dans la société française le nombre et les droits des institutrices publiques. Premier corps professionnel féminin de la nation par leurs effectifs, elles ont aussi bénéficié plus tôt que les autres de l'égalité de traitement à travail égal, et aussi du droit de vote aux élections des conseils représentatifs du personnel. Plus récemment, Mona Siegel a montré que les progrès du féminisme parmi les institutrices ont convergé avec les efforts des syn-

maîtresses était considéré par les autorités comme préoccupant - s'est opposé au projet sous prétexte qu' "il est impossible d'enseigner une matière, quelle qu'elle soit, de la même façon aux filles et aux garçons ». « Vouloir former les facultés de la femme de la même façon que les facultés de l'homme, c'est aller contre l'expérience, contre le bon sens et contre l'opinion de tous les psychologues », déclarait-il le 7 février, ce qui lui attira cette réplique de Bénassy, député socialiste d'Aubusson: "Pourquoi faites-vous enseigner le catéchisme par un curé et non par une sœur? » (Ibid.)

(1) F. Mayeur, L'enseignement secondaire des jeunes filles sous la Troisième République, Paris, Presses de la FNSP, 1977, pp. 439-440. Voir aussi les remarques de Monique Hirschhorn sur « la féminisation » dans L'ère des enseignants, Paris, PUF, 1993, pp. 121-125.

(2) Anne-Marie Sohn, Féminisme et syndicalisme: les institutrices de la Fédération unitaire de l'enseignement de 1919 à 1935, thèse de $3^{\mathrm{e}}$ cycle sous la direction d'A. Kriegel, université Paris-X, 1971.

(3) De M. Ozouf, voir en dernier lieu sur ce point Les mots des femmes. Essai sur la singularité française, Paris, Fayard, 1995.

(4) Christine Bard, Les filles de Marianne. Histoire des féminismes, 1914-1940, Paris, Fayard, 1995.

(5) Siân Reynolds, France Between the Wars: Gender and Politics, London/New York, Routledge, 1996. 
dicalistes et des pacifistes pour aboutir à ce qu'elle a appelé le « désarmement moral de la France » entre les deux guerres. Plus nombreuses à enseigner à des petits garçons, à exercer sur eux une autorité maternelle prompte à mettre l'accent sur les misères de la guerre et à leur rendre la paix désirable comme le premier des biens, elles l'étaient aussi à revendiquer des droits nouveaux pour les femmes, et cela ne pouvait manquer de donner à la première éducation civique une coloration nouvelle (1).

Cette consolidation de la place et des droits des femmes dans le corps enseignant primaire a pu contribuer à faire considérer celui-ci comme « plus homogène après qu'avant la guerre (2) », puisque le retard de la scolarisation des filles avait été comblé, les écarts de statut et de traitement réduits, et que le syndicalisme y faisait des progrès constants. L'image du «poste double » reste sans doute l'une des plus symboliques de cet âge de l'école républicaine, dans la France des bourgs et des petites villes - un âge si souvent regardé avec nostalgie de nos jours.

Or les inspecteurs et les administrateurs de l'instruction publique avaient leurs propres nostalgies, dans les années 1920 et 1930. Beaucoup regrettaient le temps où il n'était pas besoin de nommer des femmes adjointes dans les écoles de garçons, le temps où il se trouvait assez de jeunes instituteurs pour aller exercer dans les plus modestes villages. Ce n'est donc pas sans regret qu'ils ont observé la montée inexorable de la «vague féminine ». Ces réserves, ces réticences ne doivent pas être oubliées lorsqu'on évoque les droits nouveaux reconnus aux institutrices après la guerre. Ne convient-il pas de les mettre en relation avec la méfiance persistante du personnel politique pour l'octroi aux femmes du droit de vote? Ce que ces « filles de Marianne » ont en somme le mieux enseigné par l'exemple, c'est encore la patience.

Jean-François CHANET Université Charles de Gaulle - Lille 3

UMR 8529 IRHiS

(1) Mona L. Siegel, The Moral Disarmament of France. Education, Pacifism, and Patriotism, 1914-1940, Cambridge, Cambridge University Press, 2004, notamment le chapitre 3, pp. 92-122.

(2) Antoine Prost, Histoire de l'enseignement en France, 1800-1967, Paris, Armand Colin, 1968, p. 378. 